\title{
What is the Right Model for Wireless Channel Interference?
}

\author{
Aravind Iyer, Catherine Rosenberg and Aditya Karnik
}

\begin{abstract}
In wireless communications, the desired wireless signal is typically decoded by treating the sum of all the other ongoing signal transmissions as noise. In the networking literature, this phenomenon is typically abstracted using a wireless channel interference model. The level of detail in the interference model, evidently determines the accuracy of the results based upon the model. Several works in the networking literature have made use of simplistic interference models, e.g., fixed ranges for communication and interference, the capture threshold model (used in the $n s 2$ network simulator), the protocol model, and so on. At the same time, fairly complex interference models such as those based on the SINR (signal-to-interference-and-noise ratio) have also been proposed and used. We investigate the impact of the choice of the interference model, on the conclusions that can be drawn regarding the performance of wireless networks, by comparing different wireless interference models. We find that both in the case of random access networks, as well as in the case of scheduled networks (where node transmissions are scheduled to be completely conflict-free), different interference models can produce significantly different results. Therefore, a lot of caution should be exercised before accepting or interpreting results based on simplified interference models. Further, we feel that an SINR-based model is the minimum level of detail that should be employed to model wireless channel interference in a networking context.
\end{abstract}

Index Terms-Channel Modeling, interference, SINR, conflict graphs, conflict sets.

\section{INTRODUCTION}

A NY wireless signal is radiated into space by the transmitter, gets subjected to some attenuation over distance, and is finally received at the receiver, as a distorted version of itself, superposed with other wireless signals transmitted in the vicinity. Subsequently, the signal is decoded by treating the sum of all the other on-going signal transmissions as noise. Decoding success is a random event whose probability depends upon the desired signal strength, the level of thermal noise, and the strength of interfering signals. All of these phenomena have to be modeled with appropriate

Manuscript received June 2, 2008; revised September 4, 2008 and November 21, 2008; accepted December 11, 2008. The associate editor coordinating the review of this paper and approving it for publication was Dr. C.-C. Chong.

A. Iyer and A. Karnik are with the General Motors India Science Laboratory, Bangalore, 560 066, India. (e-mail: \{aravind.iyer, aditya.karnik\}@gm.com)

C. Rosenberg is with the Department of Electrical and Computer Engineering, University of Waterloo, Waterloo, ON, N2L 3G1, Canada. (e-mail: cath@ece.uwaterloo.ca)

This work was done while A. Iyer was at Purdue University, and a visiting scholar at the University of Waterloo, and while A. Karnik was at the University of Waterloo. It was supported in part by NSERC, Bell University Laboratories (BUL) and Nortel Networks. A shorter version of the paper appeared at the Third International Conference on Quality of Service in Heterogeneous Wired/Wireless Networks (QShine 2006).

Digital Object Identifier 10.1109/TWC.2009.080720. accuracy, from a networking perspective, in order to analyse and optimize the performance of wireless networks, and to propose efficient network protocols (e.g., routing). Towards this end, the networking community has employed a variety of abstractions to model wireless channel interference. Our aim, in this paper, is to ask the question: what is the right model for wireless channel interference, from a networking standpoint? Specifically, we would like to investigate the impact of the choice of the interference model, on the predicted performance of multi-access networks such as ad hoc, sensor and mesh networks.

On examining the various models for wireless channel interference extant in the literature, it is clear that interference models have indeed evolved in terms of complexity and sophistication. Starting from one of the simplest models, the co-located network à la Bianchi [1], models such as those based on the assumption of fixed communication and interference ranges, and those attempting to model "capture" (e.g., the capture threshold model used in the network simulator $n s 2$ [12]) have been proposed and used. Unlike the capture threshold model which inaccurately compares the desired signal strength with only one interfering signal at a time, a more realistic model for packet capture is used in the physical model of Gupta and Kumar [4]. Thereby, a wireless signal transmission is successful, provided the SINR perceived by the receiver, exceeds an SINR-threshold for the entire duration of the signal transmission. The SINR accounts for the cumulative interference power, and is a more natural metric for deciding packet decoding success, than the ratio of the desired signal strength to individual interference powers used in the capture threshold model.

Although wireless interference models have evolved in terms of complexity and sophistication, simplistic interference models have continued to be extensively used by the networking community, without validation. In particular, inspite of its simplistic wireless interference model, the network simulator $n s 2$ continues to find widespread use to evaluate new proposals for routing and medium access control (MAC) protocols. Further, under models which do not account for interference power additively, any two links either interfere with each other, or they can be active simultaneously, regardless of the other on-going signal transmissions. This is clearly not true in practice. Such models are currently the subject of analytical activity (for example, see [2], [7]). These works are important as stepping stones. However, their validity needs to be examined carefully before putting them to use.

Our aim is to understand which is the right model for wireless channel interference, and how accurate are existing 
simplified models, from a networking standpoint. Note that our main focus is not on modeling signal propagation on the wireless channel, but rather on modeling interference. In particular, we examine the capture threshold model, and what we term an 'interference range' model, by comparing them with a model based on SINR thresholding to model capture, with additive (rather than single) interference calculation.

We consider three case-studies. First in Section V, we consider the capacity (or max-min throughput as formulated in [8]) of a scheduled grid mesh network as a function of the number of nodes. We find that although all models predict the same order of growth for capacity at high transmit powers, their predictions are significantly different at very low transmit powers. Next in Section VI, we consider the variation of this capacity as a function of transmit power. In [8], we proved that, under the SINR based additive interference model, if the transmit powers of all the nodes in the network are scaled up by the same factor, the capacity cannot decrease. Under the interference range model, capacity could decrease with increasing power, as we find in a numerical example. Finally in Section VII, we use a discrete-event simulator to simulate different MAC protocols under the additive and the capture threshold interference models. We find that not only does the capture threshold model overestimate the throughput performance, but it also predicts different qualitative behavior.

The following are the contributions of this work:

C1 We critically study different interference models, and point out how they differ in terms of the conflict relationships they produce, and in terms of their minimum interference range.

C2 Based on the three case-studies we consider, we show that caution should be exercised before accepting results based on simplified interference models.

C3 Finally, since we believe that the impact of cumulative interference has to be taken into account in order to obtain accurate results and insights about the performance of wireless networks, we recommend the use of an additive interference model.

In the remainder, we start with a survey of related work in Section II, followed by an introduction and comparison of the wireless channel interference models we investigate, in Sections III and IV, respectively. In Sections V, VI and VII, we present in detail, the case-studies discussed above. Finally, we conclude the paper in Section VIII.

\section{RELATED WORK}

There has been a lot of research activity in the area of wireless multi-hop networks, over the past decade or so. Much work has been devoted to characterizing the throughput capacity and optimal operation of a multi-hop wireless network, and also on developing algorithms, and protocols to enable the same. However, the development and validation of simulation tools, and the analysis of common assumptions has received relatively less attention.

In [13], Takai et. al. investigate different models of wireless signal propagation, and the computational effect of increasingly sophisticated models, on large scale simulations of wireless networks. They argue that modeling wireless signal propagation merely as pathloss attenuation is not completely realistic. Although detailed models of signal propagation, can slow down execution times for wireless simulations, techniques such as parallel computation can help reduce the cost of computation. Unlike [13], our main focus is on modeling interference, not propagation.

In a closely related work [14], the authors investigate the effects of physical channel modeling on the performance of MAC and routing protocols. The authors compare the performance of the IEEE 802.11 MAC protocol [5] using two simulators, GloMoSim [3] and $n s 2$ [12]. The authors rightly point out that since $n s 2$ uses a simplistic capture threshold model for inferring collision or capture, it overestimates the throughput performance of IEEE 802.11. While our work supports the findings in [14], we further show that not only is the capture threshold model optimistic as compared to an additive interference model, but there are qualitative differences in the predictions of the two models, and they can result in different structural insights.

In [9], the authors take an experimental approach to evaluate the validity of some common assumptions made in the literature on wireless networks. The authors claim that several simplifying assumptions such as fixed transmission ranges, symmetric links, identical ranges for all radios, and so on, are still extant among the papers being published. Through extensive experimentation, the authors provide some recommendations for a good probabilistic signal propagation model. While such propagation models should be developed, and incorporated in simulators (such as $n s 2$ ), we show that it is also important to have an accurate model for interference calculation: specifically that the cumulative interference and noise should be used to determine capture.

In [11], [16], the authors assume a fixed communication range and a fixed interference range. In [17], the authors assume the capture threshold model of interference, and use $n s 2$ to evaluate their proposed MAC protocol. In [15], a model for calculating the interference range is introduced which improves upon the capture threshold model at lower powers by including the effect of noise. Other works like [2], [7] make use of the protocol model or a related binary interference model, although the authors of [7] do indicate how their work can be extended.

\section{MOdEls FOR WIRELESS CHANNEL INTERFERENCE}

As mentioned in the introduction, a variety of wireless interference models of varying levels of complexity have been proposed and used in the literature. However, there is actually no consensus on the nomenclature for wireless interference models in the literature. Hence, in order to be precise in our arguments, we formally introduce the models for wireless channel interference that we wish to investigate. We also provide a clear nomenclature for ease of presentation. Note that, in the remainder of the paper, we shall use the symbols $l$ and $m$ to denote links.

\section{A. Additive Interference Model}

The wireless interference model we introduce first, is based on additive interference, and SINR thresholding for correct 
packet reception (as in [4], [8]). This is motivated by the decoding strategies employed in several wireless physical layer technologies. Thereby, a wireless signal is decoded by treating the sum of all the other on-going signal transmissions and environmental disturbances, as noise. Decoding success is probabilistic, and the success or failure of a signal transmission can be expressed in terms of a bit/packet error probability which depends on the SINR ${ }^{1}$. For a certain acceptable BER, the SINR has to exceed an appropriate threshold. Thus, under the additive interference model a sufficient condition for packet reception is that the SINR remains greater than the threshold, throughout the duration of the packet transmission.

Denoting the transmit power used by the transmitter of link $l$ as $P_{l}$, the SINR perceived by the receiver of link $m, \gamma_{m}$, is given by:

$$
\gamma_{m}=\frac{G_{m_{o}, m_{d}} P_{m}}{P_{\mathcal{N}}+\sum_{l \in \mathcal{L} \backslash\{m\}} G_{l_{o}, m_{d}} P_{l}}
$$

where $G_{X, Y}$ denotes the channel gain from the point $X$ to the point $Y, l_{o}$ denotes the transmitter and $l_{d}$ the receiver of link $l$, and $P_{\mathcal{N}}$ denotes the thermal noise power in the frequency band of operation. Note that nodes are identified with their locations. The sum in the denominator is taken over all links $l \in \mathcal{L} \backslash\{m\}$ where $\mathcal{L}$ denotes the set of concurrently active links. The data-rate of link $m, c_{m}$, depends on the modulation and coding scheme used at the physical layer on link $m$. A packet reception at the data-rate $c_{m}$ is successful, provided that throughout the duration of the packet transmission:

$$
\gamma_{m} \geq \beta_{m}
$$

where $\gamma_{m}$ is the SINR defined by (1), and $\beta_{m}$ is an SINR threshold corresponding to an acceptable BER, depending on the modulation and coding scheme used by link $m$. Thus, under the additive interference model, if at any point during the packet transmission (2) does not hold, then there is a packet loss.

In order to simulate MAC protocols which employ physical carrier-sensing, we use the following model. A node located at $Y$ will sense the channel busy if

$$
\sum_{l \in \mathcal{L}} P_{l} G_{l_{o}, Y}+P_{\mathcal{N}} \geq \beta_{c s}
$$

where $\beta_{c s}$ is the carrier-sensing threshold.

\section{B. Capture Threshold Model}

The capture threshold model (used in $n s 2$ [12]) can be viewed as a simplification of the above model as described in (1-3). It makes use of three thresholds which we denote following the ns2 terminology, as RxThresh, CpThresh and CsThresh. The so-called receive threshold, RxThresh and the capture threshold CpThresh are both analogous to the SINR threshold $\beta$ described above. Here we have dropped the subscript for ease of description - the thresholds do depend

\footnotetext{
${ }^{1}$ Note that the SINR to bit error rate (BER) mapping is based on the Gaussian approximation of multi-access interference, motivated by the Central Limit Theorem. In practice, this approximation may sometimes not hold (for instance, see [10]), in which case the SINR may not be statistically sufficient to predict the BER. However, we feel that assuming BER as a function of the SINR is a reasonable practical approach, from a networking standpoint.
}

on the modulation and coding scheme used on the given link. Specifically, a packet reception on a link $m$ at the data-rate $c_{m}$ is successful, provided that during the packet transmission:

$$
\begin{aligned}
& P_{m} G_{m_{o}, m_{d}} \geq \text { RxThresh }_{m} \quad \text { and } \\
& \frac{P_{m} G_{m_{o}, m_{d}}}{P_{l} G_{l_{o}, m_{d}}} \geq \text { CpThresh }_{m} \quad \forall l \in \mathcal{L} \backslash\{m\}
\end{aligned}
$$

Hence, the interference is accounted for only one interferer at a time. For carrier-sensing, a node at $Y$ will sense the channel busy if

$$
P_{l} G_{l_{o}, Y} \geq \text { CsThresh for some active } l
$$

where CsThresh is the carrier-sensing threshold analogous to $\beta_{c s}$ in (3).

\section{Protocol Model}

According to the protocol model [4], a packet transmission on link $m$ is successful, provided that for each link $l \in \mathcal{L} \backslash$ $\{m\}$, we have

$$
\left|l_{o}-m_{d}\right| \geq(1+\Delta)\left|m_{o}-m_{d}\right| \text { and }\left|m_{o}-m_{d}\right| \leq R_{C}
$$

where $\Delta$ is a positive parameter and $R_{C}$ stands for communication range. Let us now show how the capture threshold model is equivalent to the protocol model, under isotropic path loss $\left(G_{x, y}=\left(\frac{|x-y|}{d_{0}}\right)^{-\eta}\right.$ where $d_{0}$ is a constant, and $\eta$ is the path loss exponent), and when all the nodes use the same transmit power and modulation and coding scheme. The two models can be seen to be equivalent under the following setting of parameters: (i) $\Delta=$ CpThresh $\cos ^{1 / \eta}-1$, and (ii) $R_{C}=d_{0}(P / \text { RxThresh })^{1 / \eta}$. This can be verified by equating the transmit powers in (5), substituting for $G_{\{.\}}$, and by comparing (5) with (7). Here the subscripts on $P$ and CpThresh have been dropped, since all the nodes use the same power and modulation and coding scheme.

\section{Interference Range Model}

The interference range model assumes fixed ranges for communication and interference (for example, see [11], [16]). To be precise, according to the interference range model, a packet reception on link $m$ is successful, provided that for each link $l \in \mathcal{L} \backslash\{m\}$, we have

$$
\left|l_{o}-m_{d}\right| \geq R_{I} \text { and }\left|m_{o}-m_{d}\right| \leq R_{C}
$$

where $R_{I}$ stands for interference range, and $R_{C}$ stands for communication range. It is convenient to take $R_{I}=R_{C}(1+$ $\Delta)$, to see how the interference range model can be viewed as simplification of the protocol model. The interference range model requires the interferer-receiver separation to be greater than a fixed quantity, the interference range, rather than proportional to the transmitter-receiver separation as in the protocol model.

A slightly more general version of the interference range model can be viewed as a simplification of the capture threshold model described above. Using the same notation as for the capture threshold model, a packet reception on a link $m$ at the data-rate $c_{m}$ is successful, provided:

$$
\begin{aligned}
P_{m} G_{m_{o}, m_{d}} & \geq \text { RxThresh }_{m} \quad \text { and } \\
P_{l} G_{l_{o}, m_{d}} & \leq \text { IrThresh }_{m} \quad \forall l \in \mathcal{L} \backslash\{m\}
\end{aligned}
$$


where IrThresh $\mathrm{I}_{m}$ is an interference threshold. By setting IrThresh ${ }_{m}=\frac{\text { RxThresh }}{\text { CpThresh }}$, one can see how the general form of the interference range model is a simplification of the capture threshold model. The interference range model requires the interfering signal strength to be smaller than a fixed quantity, rather than proportionally smaller than the received signal strength. For carrier-sensing, a node at $Y$ will sense the channel busy if

$$
P_{l} G_{l_{o}, Y} \geq \text { CsThresh for some active } l
$$

Now, the general form of the interference range model (1011) reduces to the simpler form (8), under isotropic pathloss, and when all the nodes use the same transmit power and modulation and coding scheme. The models are equivalent provided, $R_{C}=d_{0}(P / \text { RxThresh })^{1 / \eta}, R_{I}=(1+\Delta) R_{C}$, and $\Delta=$ CpThresh $^{1 / \eta}-1$.

\section{CONFlict RElationships Under DifFERENT INTERFERENCE MODELS}

We now try to understand the differences in the conflict relationships imposed by the different interference models. By conflict relationships, we mean how an individual link experiences interference (or conflict) from other individual links or from sets of other links. It is useful to characterize conflict relationships in two ways, viz., the extent of the conflict and the nature of the conflict. The extent of the conflict is merely how two links in isolation affect the operation of each other. The nature of the conflict refers to the manner in which conflict or interference from different links together affects the operation of any given link.

To illustrate the differences in the extent of conflict under the different models, we use the notion of minimum interference range. In order to define this notion, we need to make some simplifying assumptions. We assume isotropic path loss, i.e., $G_{x, y}=\left(\frac{|x-y|}{d_{0}}\right)^{-\eta}$. Let all the nodes use a power $P$ and the same modulation and coding scheme with an SINR threshold $\beta$. Under these assumptions, we define the minimum interference range to be the minimum distance from the receiver of any active link, at which a single interfering node would not cause a collision. For an active link $m$, and an interfering link $l$, the minimum interference range would be the least value of $\left|l_{o}-m_{d}\right|$ at which $l$ would not interfere with $m$ if both are concurrently active.

Under the capture threshold model, as can be seen from (5) and (7), the minimum interference range is just CpThresh $\mathrm{Ch}^{1 / \eta}$ times the transmitter-receiver separation, independent of the transmit power level. Under the additive interference model, it can be calculated using (1-2), by considering the interference from a single link as follows:

$$
\left|l_{o}-m_{d}\right| \geq\left(\frac{1}{\beta\left|m_{o}-m_{d}\right|^{\eta}}-\frac{P_{\mathcal{N}}}{P d_{0}^{\eta}}\right)^{-1 / \eta}
$$

which is evidently not a linear function of the transmitterreceiver separation. Under the interference range model, the minimum interference range is just $\mathrm{CpThresh}{ }^{1 / \eta}$ times the communication range $R_{C}$, as we saw earlier, independent of the transmitter-receiver separation. Figure 1 shows an illustration for $\eta=4$. The channel attenuation and overall noise power

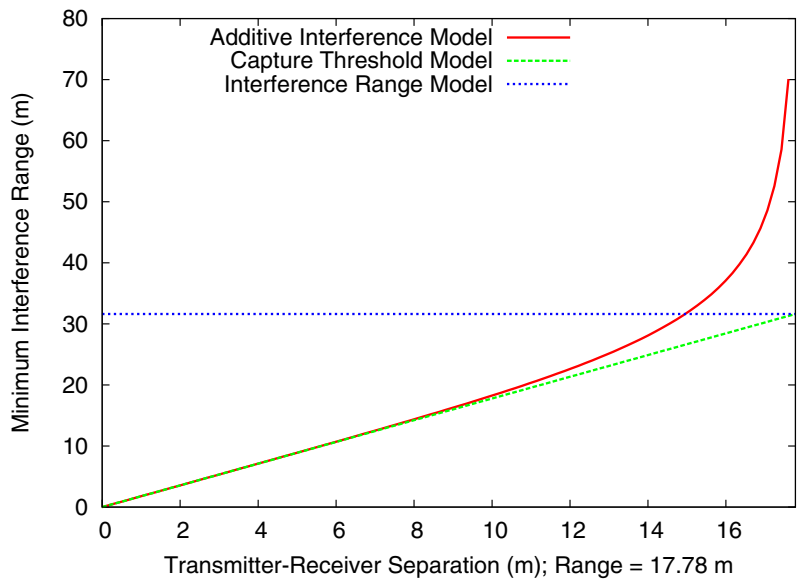

Fig. 1. Minimum Interference Range vs. Transmitter-Receiver separation

are taken from Table I which summarizes the parameters used in the paper. We consider a transmit power level of $0 \mathrm{dBm}$ and a modulation and coding scheme, with an SINR threshold of $10 \mathrm{~dB}$, and data-rate of unity. For the capture threshold model and interference range models, we take CpThresh to be identical to the SINR threshold, i.e., $10 \mathrm{~dB}$, and RxThresh to be higher than the noise power $P_{\mathcal{N}}$ by $10 \mathrm{~dB}$ (the SINR threshold).

As can be seen from Figure 1, the minimum interference range predicted by the capture threshold model is quite close to that under the additive interference model when the transmitter is close enough to the receiver. However, as the transmitterreceiver separation increases, approximating the minimum interference range as a multiple of the transmitter-receiver separation, is no longer accurate. In particular, for very "weak" links (i.e., when the receiver can just barely decode the signal from the transmitter), the minimum interference range can be arbitrarily high. The interference range model is of course, simplistic, with a constant minimum interference range for all values of the transmitter-receiver separation.

The main reason for this difference in the trend of minimum interference range, is the manner in which interference power is treated by the different models. In the additive interference model, the SINR is the primary quantity of interest, whereby the desired signal strength is compared to the sum of the noise and cumulative interference powers. In the capture threshold model, the desired signal strength is compared to RxThresh and to individual interference terms, separately. This leads to a minimum interference range that is merely proportional to the transmitter-receiver separation for the capture threshold model, but one that increases steeply close to the communication range in the case of the additive interference model, since the sum of the noise power and all the interference terms is used.

Another important difference between the interference models presented earlier, is the nature (rather than extent) of conflict relationships. Specifically, the capture threshold model, the protocol model and the interference range model, lead to binary or pair-wise conflict relationships among the wireless links (i.e., any two links either interfere with each other, or they can be active simultaneously, regardless of the other on- 
TABLE I

Physical Channel Parameters

\begin{tabular}{|l||l|}
\hline Parameters & Values \\
\hline \hline Overall Channel Bandwidth & $22 \mathrm{MHz}$ \\
\hline Overall Transmit Power & $0 \mathrm{dBm}(1 \mathrm{~mW})$ \\
\hline $\begin{array}{l}\text { Signal Attenuation (in } \mathrm{dB}) \\
\text { as a function of distance } d\end{array}$ & $-40 \mathrm{~dB}-40 \log (d)$ \\
Here $\eta$ is 4 \\
\hline Overall Noise Power & $-100 \mathrm{dBm}$ \\
\hline Carrier-sensing Threshold & $6 \mathrm{~dB}$ over noise power \\
\hline SINR Threshold for 1 Mbps & $12 \mathrm{~dB}$ \\
\hline SINR Threshold for 2 Mbps & $15 \mathrm{~dB}$ \\
\hline Busy-tone Channel Bandwidth & $11 \mathrm{kHz}$ \\
\hline $\begin{array}{l}\text { Control Channel Bandwidth } \\
\text { in DUCHA }\end{array}$ & $6.6 \mathrm{Mhz}(30 \%)$ \\
\hline
\end{tabular}

going signal transmissions). On the other hand, under the additive interference model, even though each link in a set of links may not interfere with a given link, they could still collectively cause a collision. Thus, it is incorrect to refer to the conflict relationships between just a link $l$ and a link $m$ in isolation. Rather, as we saw in [8], it is possible to enumerate the conflict sets of link $l$ which are all possible sets of links which by being active simultaneously, could cause a transmission on link $l$ to fail. This is because the additive interference model is the only model (among the ones we consider) which treats interference additively. These differences in the conflict relationships between the wireless links, will play out in significant ways in predicting capacity, performance, and so on.

\section{CASE-STUdy 1: CAPACITY AS A FUnCTION OF NUMBER OF NODES}

We consider the problem of computing the capacity of a scheduled wireless network which we explored in detail in [8]. We reproduce the formulation here for convenience. Given a wireless network that is specified in terms of the set of nodes numbered $1, \ldots, N$, a set of flows $\mathcal{F}$ numbered $1, \ldots, M$, and a set of links numbered $1, \ldots, L$, the problem of determining the capacity (or max-min throughput) of the given wireless network can be posed as a problem of optimally scheduling the independent sets in the given network. An independent set $k$ is a set of wireless links that can all be simultaneously operational without packet decoding failures in accordance with the physical parameters and SINR thresholds of all the links. $\mathcal{I}$ is used to denote the set of all independent sets in the network, and for $k \in \mathcal{I}, \alpha_{k}$ denotes the fraction of time the independent set $k$ is chosen to be active (i.e., all of its constituent links are operational). Then, the capacity of the network is the solution of the following problem:

$$
\begin{aligned}
& \max \lambda \\
& \sum_{l \in \mathcal{L}_{i}^{o}} x_{l}^{f}-\sum_{l \in \mathcal{L}_{i}^{I}} x_{l}^{f}= \begin{cases}0 & i \notin\left\{f_{s}, f_{d}\right\} \\
\lambda_{f} & i=f_{s} \\
-\lambda_{f} & i=f_{d}\end{cases} \\
& \sum_{f \in \mathcal{F}} x_{l}^{f} \leq c_{l} \sum_{k \in I_{l}} \alpha_{k} \quad l=1, \ldots, L \\
& \sum_{k \in \mathcal{I}} \alpha_{k}=1 \\
& 0 \leq \lambda \leq \lambda_{f} \quad f=1, \ldots, M
\end{aligned}
$$

where $\lambda$ is the minimum of all the flow throughputs $\lambda_{f}, x_{l}^{f}$ denotes the fraction of traffic from flow $f$ transported by link $l, f_{s}$ and $f_{d}$ respectively denote the source and destination nodes of flow $f, \mathcal{L}_{i}^{O}$ and $\mathcal{L}_{i}^{I}$ respectively denote the sets of links outgoing and incoming to node $i$, and $\mathcal{I}_{l}$ is the set of independent sets containing link $l$.

Next, we examine the capacity of a grid mesh network with a single gateway located in one corner (like the one in Figure 5 ), as a function of the number of nodes. For concreteness, let us consider an $N=n^{2}$ node network with one gateway and $N-1$ mesh nodes. Let the distance between adjacent nodes in the grid be $d$, and let $\beta$ be the SINR threshold corresponding to the lowest rate modulation and coding scheme available. For the capture threshold and interference range models, we use RxThresh and CpThresh without subscripts to denote the receive and capture thresholds, respectively, for the lowest rate modulation and coding scheme. As before, we take $\beta=$ CpThresh, and RxThresh $=\beta P_{\mathcal{N}}$. For convenience, we also take the data-rate of the modulation and coding scheme to be unity. Finally, let us assume that the channel gains are modeled by isotropic pathloss viz., $G_{x, y}=\left(\frac{|x-y|}{d_{0}}\right)^{-\eta}$. Consider the problem formulation in (13) for this single gateway mesh network. Consider one flow associated with every node (other than the gateway) destined to the gateway. Note that in the remainder of the paper, a single gateway mesh network is understood to have flows from each node to the gateway. Let 0 denote the gateway node. Then we have the following proposition.

Proposition 5.1: The throughput of a single gateway $N$ node mesh network is upper bounded by $c /(N-1)$ where $c$ is the highest operating data-rate.

Proof: See Appendix IX.

The proof is quite straightforward, and the only fact it uses is that the gateway can only receive from one node at a time. This capacity can be achieved, for example, if all the nodes communicate directly with the gateway at the highest datarate. It should therefore be clear that this conclusion hold regardless of the physical layer parameters, capabilities or even model.

However, when we look at the capacity at the lowest transmit power guaranteeing connectivity, we get different results for different models. For the capture threshold and interference range models, the capacity at low powers is considerably higher than that predicted by the additive interference model. Based on the parameters assumed, if each node uses a transmit power $P=\left(\frac{d}{d_{0}}\right)^{\eta} \beta P_{\mathcal{N}}$, then the network would be connected, since each node would be able to communicate with its adjacent nodes. The grid network is special in that at the lowest power ensuring connectivity, all the links in the network get formed simultaneously.

Proposition 5.2: Under the capture threshold and interference range models, it is possible to construct a routing and scheduling scheme which ensures a throughput lower-bounded by $\kappa / N$ for an $N$ node single gateway mesh network as described earlier, when all the nodes use the minimum power necessary for connectivity, viz., $P=\left(\frac{d}{d_{0}}\right)^{\eta} \beta P_{\mathcal{N}}$. Here $\kappa$ is a constant less than unity, independent of $N$.

Proof: See Appendix IX.

Thus, the capacity of an $N$ node grid mesh network is $\Theta(1 / N)$ for all transmit powers under the capture threshold and interference range models. In other words, the transmit 
power has no impact on the capacity, in an order sense. Let us try to understand the reason behind this result. Under both the capture threshold and interference range models which are non-additive, the success of a wireless link transmission is guaranteed, provided each concurrently active transmitter (i.e., interferer) is at least a certain distance away from the receiver of the given link, under isotropic path-loss. This distance, the minimum interference range, is upper-bounded by $R_{C}(1+\Delta)$ (refer to equations (7-8)) under both non-additive models. Owing to a bounded minimum interference range, it is possible to extract some spatial reuse (the extent of which is captured by the constant $\kappa$ ), and thus construct a schedule and routes, which can parallelize the flow of data to the gateway, efficiently enough to achieve a throughput within $\kappa$ of the upper-bound of $1 / N$. However under the additive model, in sharp contrast, the capacity at the power $P=\left(\frac{d}{d_{0}}\right)^{\eta} \beta P_{\mathcal{N}}$ is only $O\left(1 / N^{3 / 2}\right)$ as captured in the next proposition.

Proposition 5.3: Under the additive interference model, the throughput achieved by the $N$ node single gateway mesh network described earlier, at the lowest power ensuring connectivity, viz., $P=\left(\frac{d}{d_{0}}\right)^{\eta} \beta P_{\mathcal{N}}$, is $O\left(1 / N^{3 / 2}\right)$.

Proof: See Appendix IX.

The reason for this can be understood from equation (12) illustrated in Figure 1. From Figure 1, it is clear that as the transmitter-receiver separation of a link approaches the communication range $R_{C}$, the minimum interference range actually grows unbounded, under the additive interference model. Thus, at the power $P=\left(\frac{d}{d_{0}}\right) \beta P_{\mathcal{N}}$ which is just sufficient to achieve a communication range of $d$, none of the links formed in the network have any immunity to interference. Hence, they can only be scheduled one link at a time. Owing to such a restrictive amount of spatial reuse, the capacity is severely impacted to the extent that it has a different order of growth in the number of nodes.

\section{CASE-STUdy 2: CAPACity AS A FunCtion of TRANSMIT POWER}

We saw in [8] that under the additive interference model, the capacity of a wireless network does not decrease, if all the transmit powers used are scaled up by the same factor. Here we examine whether the same conclusion holds for other interference models as well. It is not hard to see that the same conclusion does hold for the capture threshold model. The reason why this is true is two-fold. Firstly, from equation (5), it is clear that by scaling up the transmit power of all the nodes by the same factor, links which could be scheduled simultaneously would remain so under the power scaling. Secondly, from equation (4), links which were not feasible earlier, could become feasible when the power is scaled up. Thus, the set of non-conflicting schedules is non-decreasing as the transmit power is scaled up, leading to the result (as also borne out by Figure 2). Note that although the throughput curves for the capture threshold model are very close to that of the additive interference model, the configurations which are optimal under the capture threshold model are often not even feasible under the more realistic conflict constraints of the additive interference model.

However, under the interference range model, this need not be true. From equation (10), by scaling up the transmit

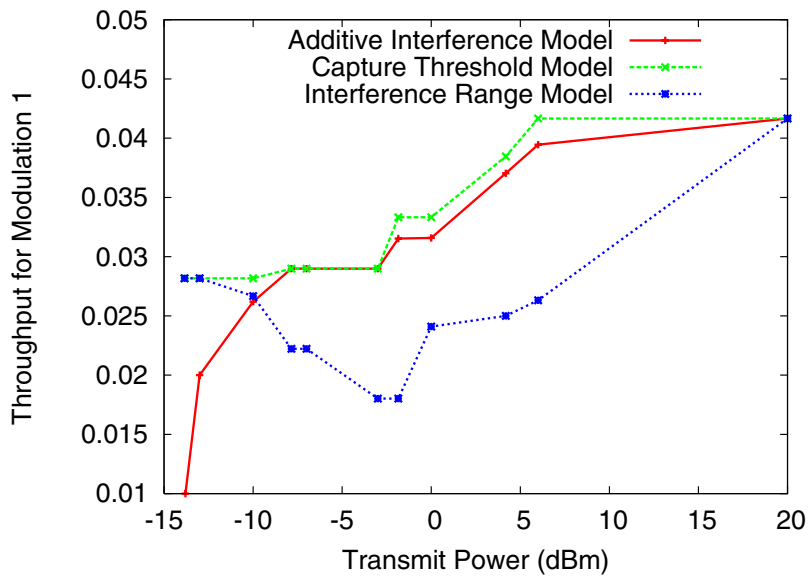

Fig. 2. Optimal Throughput vs. Transmit Power for Different Interference Models

power, the interference actually increases, thus possibly making certain independent sets conflicting under the transmit power scaling. On the other hand, from equation (10), new links could become feasible as the transmit power is scaled up. Either of these effects may dominate over the other, depending on by how much the transmit power is scaled up, the existing physical layer parameters at all the links, and so on. Consequently, the capacity predicted under the interference range model can actually reduce with increasing transmit power. This can be misleading to a network engineer dimensioning the transmit power. For example, Figure 2 shows the capacity under the interference range model decreasing for certain ranges of transmit powers, for the same network as in Figure 5.

\section{CASE-STUdy 3: MAC PROTOCOLS FOR RANDOM ACCESS WIRELESS NETWORKS}

In this section, we use a discrete-event simulator we developed, to simulate four MAC protocols, viz., IEEE 802.11 [5], RI-BTMA (receiver-initiated busy-tone multiple access) [16], DUCHA (dual channel MAC) [17] and 2CM (two channel MAC) [6]. We simulate the four protocols under both the additive and the capture threshold models of interference, and compare the throughput observed. The $2 \mathrm{CM}$ protocol has been proposed in an earlier work of ours [6]. For an introduction to the working of the four MAC protocols, the reader is directed to [6]. The values used for the simulation parameters are summarized in Table I.

We operate all the nodes with the same transmit power level (refer to Table I). For the capture threshold model, CpThresh is taken to be identical to the SINR threshold corresponding to the concerned data-rate, and RxThresh is taken to be CpThresh times the overall noise power, so that the additive and capture threshold model are consistent. The busy-tone channel bandwidth is taken to be $11 \mathrm{kHz}$. The transmit power and noise power levels are scaled down (from the values in Table I), in proportion to the bandwidth of the channel in which they are used. The carrier-sensing threshold is set to $6 \mathrm{~dB}$ over the noise power in the channel. We operate the network at the 2 Mbps data-rate. Note that we do not simulate 


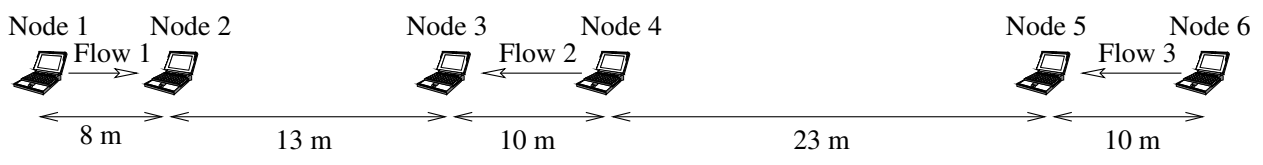

Fig. 3. A Simple Three Flow Topology

any congestion control mechanism, instead allowing the data sources to generate packets at a given rate.

The protocol DUCHA uses a separate control channel for RTS-CTS, and a data channel for DATA packets. We indicate the proportion of bandwidth used for the control channel in Table I. The rest is used for the data channel ${ }^{2}$. The IEEE 802.11 standard [5] requires that the control packets RTS and CTS, be always transmitted at the base data-rate of 1 Mbps, while the DATA and ACK packets can be transmitted at higher data-rates. We adopt the same rule for simulating the other protocols as well. To be precise, we use only the base modulation and coding scheme to transmit the RTS and CTS packets. In the case of DUCHA, the control channel datarate is always $300 \mathrm{kbps}$ ( $30 \%$ of the base data-rate, $1 \mathrm{Mbps}$ ), while the data channel data-rate is $1.4 \mathrm{Mbps}$ (70\% of the high data-rate, $2 \mathrm{Mbps}$ ).

We consider the scenario in Figure 3 with distances, node labels and flow labels, as indicated. The simulation experiment consists of simulating the network in Figure 3, with equal Poisson arrival rates for the three flows, for different arrival rates, and observing their long term throughput averaged over 1000 simulation seconds, for each value of the arrival rate. We find that the throughput values already converge around only 100 simulation seconds. The throughput achieved by Flow 3, for all the protocols, under the two interference models, is shown in Figure 4. We are interested in the highest throughput achieved by a MAC protocol while keeping the system stable. Hence we define the point of highest throughput to be the point up to which the throughput is matched by the arrival rate. Beyond this arrival rate, the arriving packets cannot be pumped out fast enough by the MAC protocol, so the link layer buffers would start to fill up, causing either buffer overflow or extremely large delays. Hence, we are interested in the portion of these graphs which is to the left of the point of maximum throughput.

There are several points to note. Firstly, note that not only does the capture threshold model overestimate the total throughput as compared to the additive interference model, but it also predicts different qualitative behavior. In particular, depending on the arrival rate, even the relative throughput rank of the protocols is different. RI-BTMA achieves the highest throughput (about $1600 \mathrm{kbits} / \mathrm{s}$ ) under the capture threshold model, but the lowest under the additive interference model (only about $200 \mathrm{kbits} / \mathrm{s}$ ). Secondly, DATA packet collisions are inevitable under the additive interference model, while they are not observed in RI-BTMA, DUCHA and 2CM, under the capture threshold model. The reason for this can be explained as follows. First consider the capture threshold model. Each

\footnotetext{
${ }^{2}$ In the paper on DUCHA [17], the authors use about $22 \%$ of the bandwidth for the control channel for a network operating at $1 \mathrm{Mbps}$. We chose the values in Table I as they resulted in a better performance for DUCHA, in the example we considered.
}
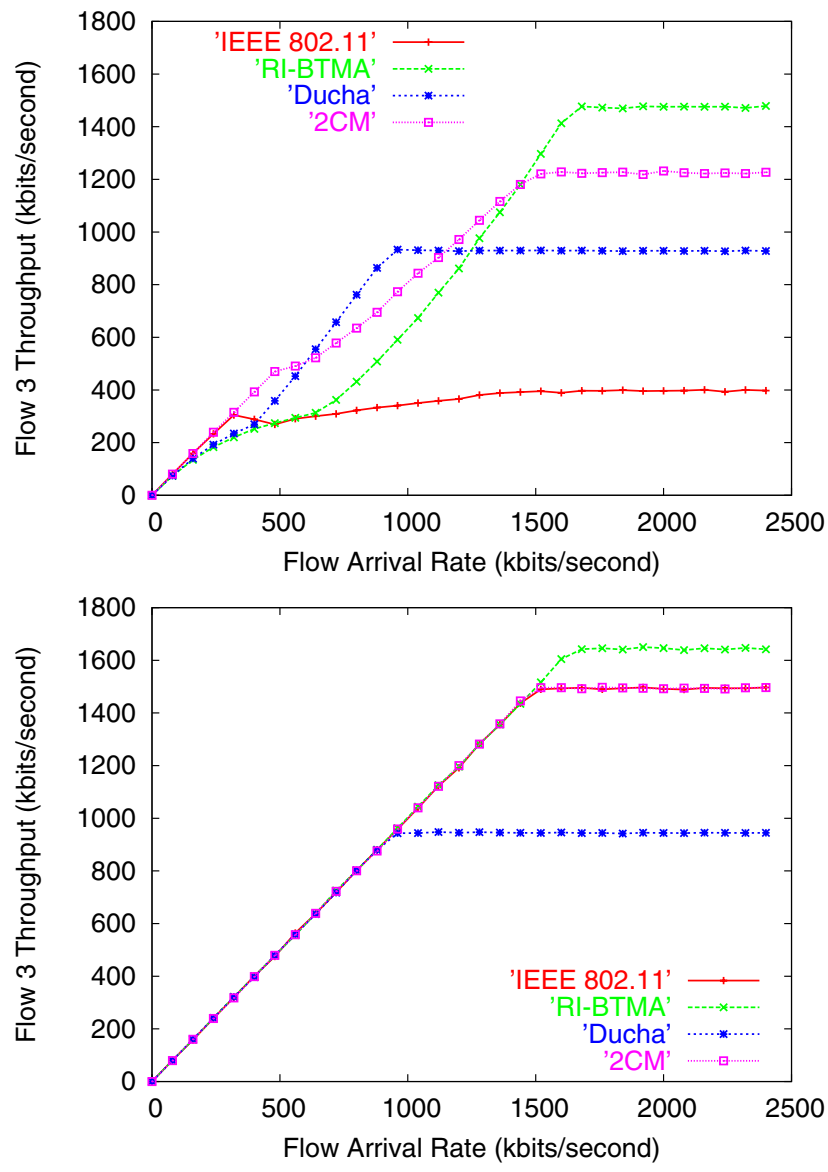

Fig. 4. Throughput of Flow 3 in Figure 3. Additive Interference model (top) and Capture Threshold Model (bottom)

of the protocols RI-BTMA, DUCHA and 2CM use a busytone signal which is asserted by the receiver for the entire duration of the DATA packet. Now, the power level of the busy-tone signal is selected in such a way that it would be received by any node within the minimum interference range of the receiver. Thus, every node which can cause a collision at the given receiver by initiating a transmission, is prevented from doing so, thereby completely preventing DATA packet collisions under all three protocols under the capture threshold model. In the case of the additive interference model, it is possible that a set of nodes, each outside the minimum interference range of the receiver, simultaneously transmit and their cumulative interference causes a collision at the given receiver.

To conclude, not only is the capture threshold model optimistic in predicting the throughput performance as compared to the additive interference model, but it also predicts different qualitative behavior, and fails to model certain phenomena which are inevitable in reality. 


\section{CONCLUSIONS}

A wireless network fundamentally differs from a wireline network in the intrinsic difficulty in abstracting its physical layer in terms of few simple per link parameters. In contrast with wired links which have fixed capacities independent of one another, in a wireless network, the links suffer from mutual interference creating a need to model the wireless channel interference. Several works in the literature have made use of simplified interference models. We have looked in detail at two such models, the capture threshold model which determines packet reception by comparing the desired signal strength with interference from a single node at a time, rather than cumulatively, and the interference range model which uses fixed ranges to model communication and interference. We have pointed out specifically how they differ in terms of the conflict relationships they produce, and in terms of their minimum interference range.

From the results of Sections V and VI it is clear that different physical layer models can lead to different results. In particular, the interference range model misleadingly predicts the capacity as decreasing with transmit power, for a range of values of the transmit power. Although, the capture threshold model predicts the capacity as non-decreasing under transmit power scaling, both the capture threshold model and the interference range model, predict a $\Theta(1 / N)$ trend for the throughput of an $N$ node grid mesh network, for all ranges of transmit powers. In sharp contrast, although it is possible to achieve the $1 / N$ bound under the additive interference model, the capacity achieved at the lowest power ensuring connectivity, for an $N$ node grid mesh network is actually $O\left(1 / N^{3 / 2}\right)$. These conflicting results clearly demonstrate the importance of using an accurate physical layer model. Further, from Section VII, we find that not only in the case of scheduled networks, but even in random access networks, these models can produce significantly different results as compared to an additive interference based model. Based on the three case-studies we have considered, it is clear that caution should be exercised before accepting results based on simplified models. Further, since we believe that the impact of cumulative interference has to be taken into account in order to obtain accurate results and insights about the performance of wireless networks, we recommend the use of an additive interference model.

Our work can be viewed as complementary to the work in [9], [13] which investigate the limitations of using isotropic path loss as a channel attenuation model. A complete model would include both realistic attenuation models, as well as accurate models for SINR calculation and determining packet reception.

\section{PROOFS}

We re-state the problem formulation we introduced in equation (13) in terms of routing variables $\phi_{f}^{r}$ instead of the flow variables $x_{l}^{f}$, since it is more instructive to express the proofs below in terms of the routing variables. Here, we assume that we have an enumeration $\mathcal{R}_{f}$ of all the feasible routes $r$ for a given flow $f$. For each $r \in \mathcal{R}_{f}$, the routing variable $\phi_{f}^{r}$ denotes the fraction of flow $f$ traffic being routed along the route $r$. The reader is referred to [8] to see that the formulation below is equivalent to the one in equation (13).

$$
\begin{array}{cl}
\max \lambda & \\
\sum_{f \in \mathcal{F}} \lambda_{f}\left(\sum_{r \in \mathcal{R}_{f}^{l}} \phi_{f}^{r}\right) \leq c_{l} \sum_{k \in \mathcal{I}_{l}} \alpha_{k} & l=1, \ldots, L \\
\sum_{r \in \mathcal{R}_{f}} \phi_{f}^{r}=1, \phi_{f}^{r} \geq 0 & f=1, \ldots, M \\
\sum_{k \in \mathcal{I}} \alpha_{k}=1 & \\
0 \leq \lambda \leq \lambda_{f} & f=1, \ldots, M
\end{array}
$$

Proof of Proposition 5.1: Since $\lambda$ is the minimum flow throughput, we have

$$
\begin{aligned}
\lambda & \leq \frac{\sum_{f \in \mathcal{F}} \lambda_{f}}{N-1}=\frac{\sum_{f \in \mathcal{F}} \sum_{l \in \mathcal{L}_{0}^{I}} x_{l}^{f}}{N-1} \\
& \leq \frac{\sum_{l \in \mathcal{L}_{0}^{I}} c_{l} \sum_{k \in \mathcal{I}_{l}} \alpha_{k}}{N-1} \\
& \leq \frac{c}{N-1} \sum_{l \in \mathcal{L}_{0}^{I}}(\text { Time-fraction } l \text { is active }) \\
& =\frac{c}{N-1}(\text { Time-fraction } 0 \text { is receiving }) \leq \frac{c}{N-1}
\end{aligned}
$$

Lemma 9.1: Consider the capture threshold model, with RxThresh and CpThresh denoting the receive and capture thresholds respectively, for the lowest rate modulation and coding scheme available. Consider two links $l$ and $m$, with $\left|l_{o}-l_{d}\right|=\left|m_{o}-m_{d}\right|=d$. Let the transmit powers on both links be $P$ such that both the links $l$ and $m$ are feasible to operate under the lowest rate modulation and coding scheme. Assume that the channel gains are only due to isotropic pathloss. To be precise, $G_{x, y}=\left(\frac{|x-y|}{d_{0}}\right)^{-\eta}$. Then, there exists an integer $k$ such that if $\left|l_{o}-m_{o}\right|=k d$, the two links can be operated simultaneously at the lowest rate modulation and coding scheme.

Proof: For the link $m$ to be operating successfully at the lowest rate modulation and coding scheme, under the capture threshold model, it is easy to see that we require:

$\frac{P\left(\frac{\left|m_{o}-m_{d}\right|}{d_{0}}\right)^{-\eta}}{P\left(\frac{\left|l_{o}-m_{d}\right|}{d_{0}}\right)^{-\eta}}>$ CpThresh $\Leftrightarrow\left|l_{o}-m_{d}\right|>$ CpThresh $^{1 / \eta} d$

Similarly, for link $l$ to be operating successfully, we require $\left|m_{o}-l_{d}\right|>$ CpThresh ${ }^{1 / \eta} d$. Now, choose $k>1+$ CpThresh ${ }^{1 / \eta}$ and let $\left|l_{o}-m_{o}\right|>k d$. It is easy to see by the triangle inequality that $\left|l_{o}-m_{d}\right|$ and $\left|m_{o}-l_{d}\right|$ are then greater than CpThresh ${ }^{1 / \eta} d$.

Lemma 9.2: Lemma 9.1 holds for the interference range model as well with parameters denoted identically.

Proof: Similar to that of Lemma 9.1 except with $k>1+$ $\left(\frac{d_{0}}{d}\right)\left(P \frac{\text { CpThresh }}{\text { RxThresh }}\right)^{1 / \eta}$.

Proof of Proposition 5.2: Let $k$ be an integer such that two links can be scheduled simultaneously, at the lowest rate modulation and coding scheme, if their transmitters are $k d$ apart from one another. The existence of such a $k$ for both the capture threshold and the interference range models, follows from Lemma 9.1 and Lemma 9.2. Let us visualize the network as indicated in Figure 5, and identify each node with its coordinates (say $(i d, j d))$. Let $\left(\left(i_{1}, j_{1}\right),\left(i_{2}, j_{2}\right)\right)$ represent a directed link from node $\left(i_{1} d, j_{1} d\right)$ to node $\left(i_{2} d, j_{2} d\right)$. Now, let 


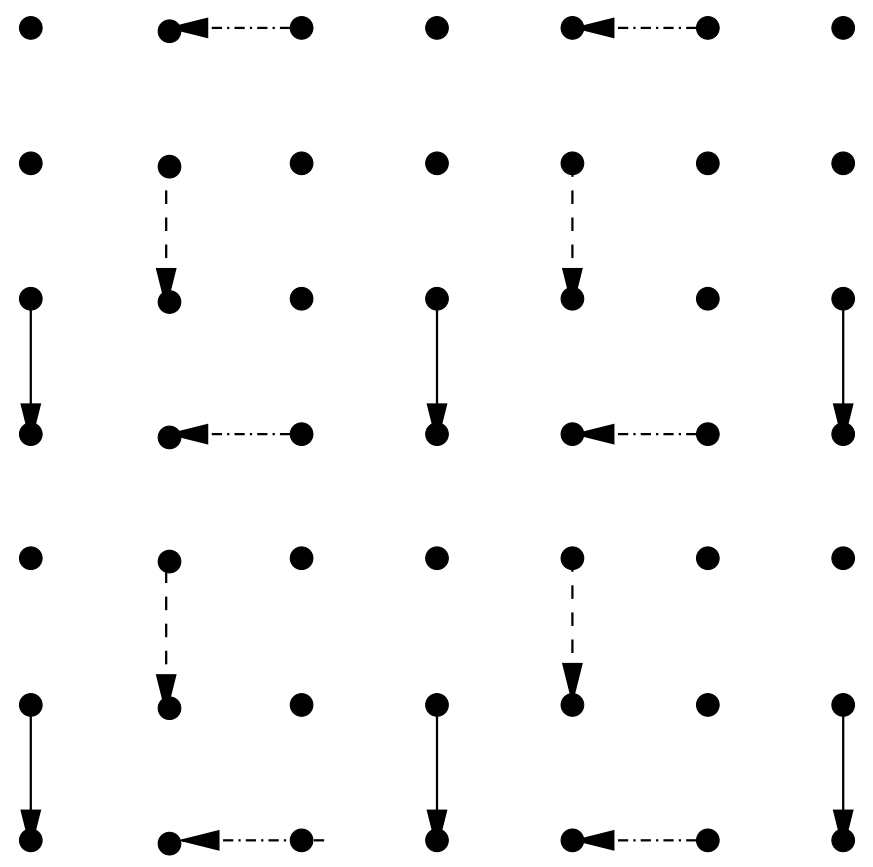

Fig. 5. Illustrating the construction of $I_{1,1,1}$ for a 49 node grid with $k=3$. Clearly, $n=7$. The solid links represent $I_{1,1,1}$ and the dashed and dot-dashed links represent its shifted and rotated versions, respectively.

us construct an independent set of downward directed links, denoted by $I_{1,1,1}$ :

$$
\left\{\begin{array}{lll}
((0,1),(0,0)), & ((k, 1),(k, 0)), & \ldots \\
((0, k+1),(0, k)), & ((k, k+1),(k, k)), & \ldots \\
((0,2 k+1),(0,2 k)), & ((k, 2 k+1),(k, 2 k)), & \ldots \\
\ldots & \cdots & \ldots \\
\ldots & \cdots & \ldots
\end{array}\right\}
$$

It is clear that $I_{1,1,1}$ is an independent set since the transmitters of all the links are a distance $k d$ apart from one another (see Figure 5). In a similar fashion, for $p=\{1,2\}$, $q=\{1,2, \ldots, k\}$ and $r=\{1,2, \ldots, k\}$, we can construct $2 k^{2}$ independent sets $I_{p, q, r}$ as follows:

$$
\begin{aligned}
I_{p, q, r}= & \{((i, j),(i+1-p, j-1+p) \\
& \text { for } i=q, q+k, q+2 k, \ldots ; \\
& \text { and } j=r, r+k, r+2 k, \ldots ; \\
& \text { such that } 1 \leq i, j \leq n \\
& \text { and } 1 \leq i+1-p, j-1+p \leq n\}
\end{aligned}
$$

Now the scheduling strategy is to schedule each of these $2 k^{2}$ independent sets for an equal fraction of time $\frac{1}{2 k^{2}}$. Under this scheduling scheme, each leftward and downward link is assigned a capacity of $\frac{1}{2 k^{2}}$ and all the other links are assigned a capacity of 0 . Now consider a routing scheme $\phi=\left(\phi_{f}^{r}\right)_{f \in \mathcal{F}, r \in \mathcal{R}_{f}}$ which uses only leftward and downward links.

Then, from the routing formulation in equation (14) on page 8 , it is clear that $\lambda$ would be a feasible solution provided $\lambda=\lambda_{f}$ for all $f \in \mathcal{F}$, and provided for every link $l$, we have:

$$
\lambda\left(\sum_{f \in \mathcal{F}} \sum_{r \in \mathcal{R}_{f}^{l}} \phi_{f}^{r}\right) \leq \frac{1}{2 k^{2}}
$$

In particular,

$$
\lambda=\hat{\lambda}:=\frac{1}{2 k^{2}} \min \left\{\frac{1}{\sum_{f \in \mathcal{F}} \sum_{r \in \mathcal{R}_{f}^{l}} \phi_{f}^{r}}\right\}
$$

is a feasible solution. Further, we have that

$$
\hat{\lambda} \geq \frac{1}{2 k^{2}} \frac{1}{\sum_{f \in \mathcal{F}} \sum_{r \in \mathcal{R}_{f}} \phi_{f}^{r}}>\frac{1}{2 k^{2} N}
$$

thus, proving the proposition with $\kappa=\frac{1}{2 k^{2}}$.

Proof of Proposition 5.3: Since all the nodes operate with a power $P=\left(\frac{d}{d_{0}}\right)^{\eta} \beta P_{\mathcal{N}}$, the SINR for any active link $l$ would be given by:

$$
\frac{\left(\frac{d}{d_{0}}\right)^{\eta} \beta P_{\mathcal{N}} G_{l_{o}, l_{d}}}{P_{\mathcal{N}}+\sum_{l^{\prime} \neq l}\left(\frac{d}{d_{0}}\right)^{\eta} \beta P_{\mathcal{N}} G_{l^{\prime}{ }_{o}, l_{d}}}=\frac{\beta}{1+\sum_{l^{\prime} \neq l} \beta} \leq \beta
$$

where the summation is over all the active links $l^{\prime} \neq l$. Clearly, equality is achieved when the link $l$ is the only link active. With slight abuse of the notation, let us denote the independent set containing link $l$ also by $l$, and let $\alpha_{l}$ be the time fraction for which it is active. Then, we have from the routing formulation in equation (14):

$$
\sum_{f \in \mathcal{F}} \lambda_{f}\left(\sum_{r \in \mathcal{R}_{f}^{l}} \phi_{f}^{r}\right) \leq \alpha_{l} l=1, \ldots, L
$$

where the right-hand side is simply $\alpha_{l}$ since the data-rate corresponding to the lowest rate modulation and coding scheme, is taken to be unity.

$$
\begin{gathered}
\Rightarrow \sum_{l \in \mathcal{L}} \sum_{f \in \mathcal{F}} \lambda_{f}\left(\sum_{r \in \mathcal{R}_{f}^{l}} \phi_{f}^{r}\right) \leq \sum_{l \in \mathcal{L}} \alpha_{l}=1 \\
\Rightarrow \sum_{f \in \mathcal{F}} \lambda_{f}\left(\sum_{r \in \mathcal{R}_{f}} h_{r} \phi_{f}^{r}\right) \leq 1
\end{gathered}
$$

where $h_{r}$ is the number of hops of route $r$. For flow $f$, let $h_{f}^{\min }$ be the minimum number of hops of any route in $\mathcal{R}_{f}$. Using the fact that $\sum_{r \in \mathcal{R}_{f}} \phi_{f}^{r}=1$, we have:

$$
\lambda \leq \frac{1}{\sum_{f \in \mathcal{F}} h_{f}^{\min }}
$$

It is easy to see that the flow associated with the node at $(i d, j d)$ has to travel at least $i+j$ hops to reach the gateway. Thus, we have

$$
\lambda \leq \frac{1}{\sum_{i=0, j=0}^{n-1, n-1}(i+j)} \Rightarrow \lambda=O\left(n^{3}\right)=O\left(N^{\frac{3}{2}}\right)
$$

since $N=n^{2}$, and that proves the proposition.

\section{REFERENCES}

[1] G. Bianchi. "IEEE 802.11 - Saturation throughput analysis," IEEE Commun. Lett., vol. 2, no. 12, Dec. 1998.

[2] L. Chen, S. H. Low, and J. C. Doyle. "Joint congestion control and media access control design for ad hoc wireless networks," in Proc. IEEE INFOCOM 2005.

[3] "The GloMoSim network simulator," [Online]. Available: http://pcl.cs.ucla.edu/projects/glomosim/

[4] P. Gupta and P. R. Kumar. "The capacity of wireless networks," IEEE Trans. Inform. Theory, vol. IT-46, no. 2, pp. 388-404, Mar. 2000. 
[5] IEEE 802 LAN/MAN Standards Committee. "Wireless lan medium access control (MAC) and physical layer (PHY) Specifications," IEEE Std. 802.11-1999 Edition, 1999.

[6] A. Iyer and C. Rosenberg, "Understanding the key performance issues with MAC protocols for multi-hop wireless networks," Wiley J. Wireless Commun. Mobile Computing, Special Issue MAC Protocols Wireless Ad Hoc Networks," Aug. 2007.

[7] K. Jain, J. Padhye, V. N. Padmanabhan, and L. Qiu. Impact of interference on multi-hop wireless network performance. In Proceedings of the ACM/IEEE MobiCom 2003, 2003.

[8] A. Karnik, A. Iyer and C. Rosenberg, "Throughput-optimal configuration of wireless networks," IEEE/ACM Trans. Networking, to appear.

[9] D. Kotz, C. Newport, R. Gray, J. Liu, Y. Yuan, and C. Elliott, "Experimental evaluation of wireless simulation assumptions," in Proc. ACM MSWiM, 2004.

[10] A.-M. Mourad, A. Guèguen and R. Pyndiah. "MAI analysis for forward link mono-dimensionally spread OFDM systems," in Proc. IEEE VTC, Spring 2004.

[11] T. Nandagopal, T-E. Kim, X. Gao, and V. Bharghavan. "Achieving mac layer fairness in wireless packet networks," In Proceedings of the ACM/IEEE MobiCom 2000, August 2000.

[12] "The ns2 network simulator," [online]. Available: http://www.isi.edu/nsnam/ns/

[13] M. Takai, R. Bagrodia, A. Lee, and M. Gerla, "Impact of channel models on simulation of large scale wireless networks," in Proc. $2^{\text {nd }} A C M$ International Workshop Modeling, Analysis Simulation Wireless Mobile Systems, MSWiM '99.

[14] M. Takai, J. Martin and R. Bagrodia, "Effects of wireless physical layer modeling in mobile ad hoc networks," in Proc. $2^{\text {nd }}$ ACM International Symposium Mobile Ad Hoc Networking Computing, MobiHoc, 2001.

[15] A. T. Toyserkani, E. G. Strom and A. Svennson, "Improved model for transmission and interference ranges," Technical Report 42050. Series $R$ - Department Signals Systems, Chalmers University of Technology, Göteborg, 2007.

[16] C. Wu and V. O. K. Li. "Receiver-initiated busy-tone multiple access in packet radio networks," in Proc. ACM SIGCOMM '87, pp.s 336-342, 1987.

[17] H. Zhai, J. Wang, Y. Fang, and D. Wu. "A dual-channel mac protocol for mobile ad hoc networks," in Proc. IEEE GLOBECOM 2004, Workshop Wireless Ad Hoc Sensor Networks, Nov. 2004.

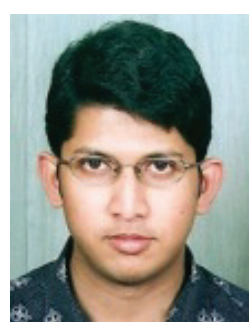

Aravind Iyer received his B.Tech. and M.Tech. degrees in Electrical Engineering, from the Indian Institute of Technology (IIT) Bombay in 2002, and his Ph.D. degree from the School of Electrical and Computer Engineering, at Purdue University, in 2007. From 2004 till 2006, he was a visiting research scholar at the Department of Electrical and Computer Engineering, University of Waterloo, Canada. Currently, he is with the Vehicular Communication and Information Management group in the General Motors India Science Laboratory, Bangalore. His research interests include the design, modeling and optimization and security of wireless networks such as vehicular wireless networks, sensor networks, ad hoc networks, and wireless mesh networks.

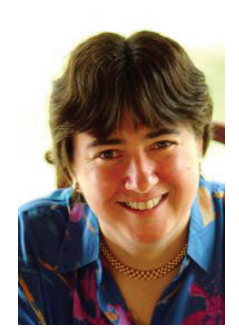

Catherine Rosenberg was born and educated in France (Ecole Nationale Supérieure des Télécommunications de Bretagne, Diplôme d'Ingénieur in EE in 1983 and University of Paris, Orsay, Doctorat en Sciences in CS in 1986) and in the USA (UCLA, MS in CS in 1984), Dr. Rosenberg has worked in several countries including USA, UK, Canada, France and India. In particular, she worked for Nortel Networks in the UK, AT \& T Bell Laboratories in the USA, Alcatel in France and taught at Purdue University (USA) and Ecole Polytechnique

of Montreal (Canada).

In 2004, Dr. Rosenberg joined the University of Waterloo where she holds a University Research Chair. She has been on the Scientific Board of France- Telecom since 2007. Her research interests are broadly in networking with currently an emphasis in wireless networking and in traffic engineering (Quality of Service, Network Design, and Routing). She has authored over 100 papers and has been awarded eight patents in the USA.

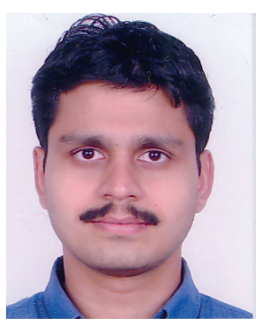

Aditya Karnik received the B.E. degree in Electronics and Telecommunications from the University of Pune, Pune, India, and the M.E. and Ph.D degree, both in Electrical Communication Engineering, from the Indian Institute of Science, Bangalore, India. During his $\mathrm{Ph} . \mathrm{D}$, he was a recipient of the IBM Research Fellowship. He is currently with the Manufacturing Enterprise Modeling group in the General Motors India Science Laboratory, Bangalore. His research interests are in control and optimization theory in general, and its application to communication and manufacturing systems in particular. 